\title{
Hygiene - gold standard not only in prevention of COVID-19 infection
}

\author{
Barbara Nieradko-Iwanicka \\ Chair and Department of Hygiene, Medical University of Lublin, Poland
}

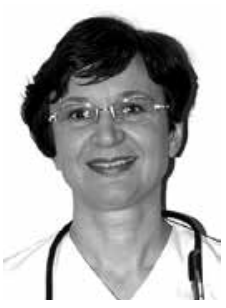

\begin{abstract}
About the Author
Barbara Nieradko-Iwanicka was born in Kraśnik and is currently associated with the Lublin medical community. She is a specialist in internal medicine and rheumatology and a professor at the Medical University of Lublin working as an assistant professor at the Chair and Department of Hygiene. Her doctoral dissertation was entitled "The effect of the combined action of plant protection products and experimental brain hypoxia on memory processes in mice" and her habilitation thesis concerned "The effect of synthetic pyrethroids administered long-term to mice after transient cerebral ischemia on their behavior, parameters of oxidative stress and the functions of selected internal organs". She is an academic teacher, a supervisor of doctoral and master's theses and a popularizer of science.
\end{abstract}

In 1847 Ignaz Semmelweis conducted the first clinical trial concerning the effect of hand hygiene on mortality rate among the patients of the obstetric clinic at the University of Vienna Allgemeine Krankenhaus. He observed that maternal mortality rates, mostly attributable to puerperal fever, were substantially higher in the clinic where medical students were trained (16\%) compared with the other (7\%) where midwives were trained [1]. Semmelweis also noted that doctors and medical students often went directly to the delivery suite after performing autopsies. He suspected presence of "cadaverous particles" on the hands despite handwashing with soap and water. Semmelweis recommended for students' and doctors' that hands should be scrubbed in a chlorinated lime solution before every patient contact and particularly after leaving the autopsy room. As a result of his efforts the mortality rate dramatically declined to $3 \%$ in this clinic [1].

Almost two centuries after Semmelweis observations, adherence to the hand hygiene standards is below $40 \%$ [2].

The modern world is frightened by data about high morbidity and mortality in the course of SARS-CoV-2 infection. The current pandemic situation has sparked a discussion on hygiene and vaccination and there is still hope for effective prevention of SARS-Cov-2 infection.
The compulsory calendar of children's immunization was introduced in Poland in 1950s, developed afterwards and since then we have lived in comfort of being protected from life-frightening infectious diseases. Herd immunity created via vaccination contributed to the eradication of some infectious diseases (e.g. smallpox, measles, whooping cough, rubella). $20^{\text {th }}$ century brought the wider use of antibiotics, reduced maternal mortality rate, free healthcare for all children. All these factors played a role in increasing lifespans of Poles. The average lifespan is 81.8 for women and 74 for men living in Poland at the beginning of the $21^{\text {st }}$ century [3].

At present the main health hazards in developed countries seem to be the non-communicable diseases (cardiovascular diseases, diabetes, obesity, neoplasms) [4]. In Poland the main causes of death are cardiovascular and cerebrovascular diseases $60.6 \%$ and $17.3 \%$ respectively [3].

The problem of the developed countries is the gradual ageing of their populations. Numerous comorbidities reduce immunity in the elderly. Anti-vaccination movements are also becoming a separate problem. Some parents avoid vaccination of their children and this plays a role in reducing the herd immunity. Lack of hygiene at healthcare setting and in everyday life enables the spread of infections especially in overcrowded areas.

Address for correspondence:

Barbara Nieradko-Iwanicka, Chair and Department of Hygiene, Medical University of Lublin, 11 Radziwiłłowska St. (Collegium Medicum), 20-080 Lublin, e-mail: bnieradkoiwanicka@wp.pl

Submitted: 29.03.2020; Accepted: 4.08.2020 
People are free to travel, gather up at overcrowded airports, marketplaces, sites of sport and cultural events. All these factors enable airborne infectious diseases like SARS-Cov-2 to spread.

During the course of education at medical schools students often neglect the subject entitled "hygiene". Many complain that it is "a waste of time". In the era of COVID-19 it makes sense to recall the rules of hand hygiene established by the World Health Organization (WHO) to be used in practice not only at the time of COVID-19 pandemic, but also universally.

In 2009 were issued WHO Guidelines on Hand Hygiene in Health Care [2]. Hand hygiene among healthcare workers is usually concerned as means to prevent cross contamination of patients and healthcare associated infections ( $\mathrm{HCAl}$ ). Healthcare associated infections implies prolonged hospital stay, long-term disability, increased resistance of microorganisms to antimicrobials, massive additional financial burden, high costs for patients and their families, and excess deaths. In developed countries, HCAl concerns 5-15\% of hospitalized patients and can affect $9-37 \%$ of those admitted to intensive care units (ICUs) [5].

The most frequent type of hospitals' infection is urinary tract infection (UTI) (36\%), followed by surgical site infection (SSI) (20\%), bloodstream infection (BSI), and pneumonia (both 11\%) [6]. In Europe reported hospital prevalence rates of patients affected by HCAl range from $4.6 \%$ to $9.3 \%$. In developing countries it is $5.7 \%$ to $19.1 \%$ [2]. In the era of COVID-19 the term "hygiene" gets a new dimension: lifesaving.

The aim of this article is to review the methods and indications for hand hygiene at health care settings and in everyday life. Documents issued by WHO are reviewed as well as publications related to the topic found in PubMed database.

There are two methods of hand hygiene: hand washing with soap and water and hand disinfection with alcohol-based formulas (containing $60-80 \%$ of ethanol, propanol or isopropanol).

When washing hands with soap and water:

- wet hands with water,

- apply the amount of soap necessary to cover all surfaces; liquid, bar, leaf or powdered forms of soap are acceptable,

- rinse hands with water, use clean, running water whenever possible; avoid using hot water, as repeated exposure to hot water may increase the risk of dermatitis,

- dry thoroughly with a single-use towel; use towel to turn off tap/faucet; make sure towels are not used multiple times or by multiple people.
Wash hands with soap and water when are:

- visibly dirty,

- contaminated with proteinaceous material, blood, or other body fluids,

- if exposure to Bacillus anthracis or Clostridium difficile is suspected or proven (since the physical action of washing and rinsing hands in such circumstances is recommended because alcohols, chlorhexidine, iodophors, and other antiseptic agents have poor activity against spores),

- after using a restroom, wash hands with soap and water,

- before and after having food.

The amount of soap used matters - it should be minimum $3 \mathrm{ml}$. In a study conducted by Larson et al. [7], the authors have found that using only $1 \mathrm{ml}$ of liquid soap or alcohol-based handrub yielded lower log reductions (greater number of bacteria remaining on hands) than using $3 \mathrm{ml}$ of the product to clean hands.

When using and alcohol-based handrub:

- apply a palmful of alcohol-based handrub and cover all surfaces of the hands, rub hands until dry,

- rub hands palm to palm,

- right palm over the left dorsum with fingers interlaced and vice versa (repeat each movement 5 times),

- palm to palm with fingers interlaced,

- backs of fingers to opposing palms with fingers interlocked,

- rotational rubbing of left thumb clasped in right hand and vice versa,

- rotational rubbing backwards and forwards with clasped fingers of right hand in left palm and vice versa.

It should take 20-30 seconds; once dry your hands are safe [2].

When hands are not visibly soiled, an alcohol-based hand rub should be used routinely for decontaminating hands:

- before having direct contact with patients,

- before donning sterile gloves when inserting a central intravascular catheter,

- before inserting indwelling urinary catheters, peripheral vascular catheters, or other invasive devices that do not require a surgical procedure,

- after contact with a patient's intact skin (when taking a pulse or blood pressure or lifting a patient),

- after contact with body fluids or excretions, mucous membranes, nonintact skin, and wound dressings if hands are not visibly soiled,

- after contact with inanimate objects (including medical equipment) in the immediate vicinity of the patient,

- after removing gloves,

- if moving from a contaminated body site to a clean body site during patient care [2]. 
Among chemicals that have proven antimicrobial efficacy are alcohols, chloroxylenol, chlorhexidine, hexachlorophene, iodophors, triclosan and quaternary ammonium compounds. All are effective against Gram positive and Gram negative bacteria [2]. Hexachlorophene and triclosan have doubtful potency against enveloped and non-enveloped viruses. Triclosan and quaternary ammonium compounds not always combat mycobacteria and fungi. Interestingly iodophores are the only chemicals that have poor activity against spores. Ultimately alcohols were chosen by the WHO as chemicals of choice for hand hygiene [2].

Unfortunately, according to statistics only $38 \%$ of healthcare workers obey the hand hygiene rules [2]. WHO recommends visual prompters placed next to each tap at healthcare settings, lectures, giving good role models by persons in charge of the wards and outpatient clinics.

In the study conducted by Ahmed et al. [8] in Karachi published in March 2020 assessing the adherence to $\mathrm{WHO}$ hand hygiene guidelines with 212 participants (74 doctors, 66 nurses, 52 technicians, and 20 ward assistants) the compliance with hand disinfectant use before and after every patient contact was $12.3 \%$. The use of disinfectant was higher among males than females (mean 7.88 times for males versus 6.20 for females) and the younger individuals were more compliant with hand hygiene practices; $62.73 \%$ of participants were aware of the WHO guidelines regarding hand hygiene and $65.56 \%$ were aware of HCAl. Half of cited study participants (45.75\%) had never attended a formal lecture on hand hygiene and $62.26 \%$ were unaware of the complications of HCAl [8].

According to Sax et al. [9] who spread a questionnaire about adherence to hand hygiene rules among 271 physicians (26\%), 629 nurses (60.4\%), 141 nursing assistants (13.5\%) and found that social pressure from patients to perform hand hygiene was ranked highly by $73.7 \%$ of respondents, pressure from superiors was ranked highly by $66.8 \%$, pressure from colleagues was ranked highly by $57.9 \%$, and pressure from the person perceived to be most influential was ranked highly by $68.8 \%$. High self-reported rates of adherence to hand hygiene (defined as performance of proper hand hygiene during $80 \%$ or more of hand hygiene opportunities) was independently associated with female gender, training in hand hygiene, participation in a previous hand hygiene campaign, peer pressure from colleagues, perceived good adherence by colleagues, and the perception that hand hygiene is relatively easy to perform [9].

$\mathrm{Kac}$ et al. [10] conducted a comparative study of microbiological efficacy of handrubbing with an alcohol-based solution and handwashing with an unmed- icated soap. The study presented that: $15 \%$ of healthcare workers hands were contaminated with transient pathogens beforehand hygiene; no transient pathogens were detected on their hands after handrubbing in majority of participants except for two cases.

In the study conducted by Gorou et al. [11] the efficacy of handrubbing with alcohol based solution versus standard handwashing with antiseptic soap was assessed in a randomised clinical trial. Twenty three healthcare workers from three intensive care units in a French university hospitals participated in the study. Handrubbing with alcohol based solution (in 12 workers) or handwashing with antiseptic soap (in 11 workers) when hand hygiene was indicated before and after patient care were performed. Imprints were taken of fingertips and palm of dominant hand before and after hand hygiene procedure. Bacterial counts were quantified blindly.

With handrubbing the median percentage reduction in bacterial contamination was significantly higher than with handwashing ( $83 \%$ vs. $58 \%$ ), with a median difference in the percentage reduction of $26 \%$. The median duration of hand hygiene was 30 seconds in each group. The authors concluded that during routine patient care handrubbing with an alcohol based solution is significantly more efficient in reducing hand contamination than handwashing with antiseptic soap [11].

Chow et al. [12] conducted a randomized controlled trial to compare the effectiveness of 3 hand hygiene protocols during routine inpatient care: handrubbing with alcohol covering all hand surfaces, handrubbing with alcohol using the standard technique, and handwashing with chlorhexidine using the standard technique. Hand samples were obtained from 60 medical and 60 nursing staff, before and after hand hygiene. Quantitative and qualitative bacterial evaluations were carried out by microbiologists blinded to the protocol. All 3 protocols were effective in reducing hand bacterial load. During routine patient care, alcohol handrubbing covering all hand surfaces required less time (median 26 seconds) than alcohol handrubbing using the standard technique (median 38.5 seconds) and chlorhexidine handwashing (median 75.5 seconds). The authors concluded that alcohol handrubbing protocols are as efficacious as chlorhexidine handwashing. Alcohol handrubbing covering all hand surfaces is the most time-effective protocol for routine patient care activities in busy general wards [12].

Many healthcare workers complain that the hand hygiene procedures take too long time. Pires et al. [13] confirmed in their study that handrubbing for $15 \mathrm{sec}$ onds was non-inferior to 30 seconds in reducing bacterial load, irrespective of type of bacteria or contamination fluid concentration. 
Repeated use of soaps and alcohol-based hand hygiene products may cause two types of skin reactions related to hand hygiene:

1) irritant contact dermatitis - the most common type includes symptoms that can vary from quite mild to debilitating, including dryness, irritation, itching, and even cracking and bleeding,

2) allergic contact dermatitis, is rare and represents an al-

lergy to some ingredient in a hand hygiene product [2].

Therefore between patients and after work skin care products should be used.

Healthcare workers have to pay a special attention to their nails, especially if they work at intensive care units or with patients with impaired immunity. Numerous studies have documented that subungual areas of the hand harbour high concentrations of bacteria, most frequently coagulase-negative staphylococci, Gram-negative rods, Corynebacteria, and yeasts. Freshly applied nail polish does not increase the number of bacteria recovered from periungual skin, but chipped nail polish may support the growth of larger numbers of organisms on fingernails. Even after careful handwashing or surgical scrubs, healthcare workers often harbour large numbers of potential pathogens in the subungual spaces. Fingernail diseases may reduce the efficacy of hand hygiene and result in the transmission of pathogens. The healthcare workers must not wear artificial fingernails or extenders when having direct contact with patients and natural nails should be kept short $(0.5 \mathrm{~cm}$ long) [2].

Healthcare workers often wear examination gloves. The gloves are indicated before [2]:

- contact with blood,

- contact with mucous membrane and non-intact skin,

- potential presence of highly infectious organism,

- in case of an epidemic,

- emergency situation,

- intravenous line insertion or removal,

- drawing blood,

- discontinuation of venous line,

- pelvic and vaginal examination,

- suctioning non-closed systems of endotracheal tubes,

- emptying emesis basins,

- handling/cleansing instruments,

- handling waste,

- cleaning up spills of body fluids.

At that moment all the public is afraid of highly contagious infectious airborne diseases. Viruses and bacteria can be spread through coughing, sneezing, laughing or through close personal contact. These pathogens ride on either dust particles or small respiratory droplets and can stay suspended in air and re capable of traveling distances on air currents. Airborne diseases are most commonly seen in unsanitary conditions and overcrowded areas. Also, these diseases easily spread in areas of poverty and poor hygienic conditions.

Therefore respiratory hygiene and ventilation are of great importance in all cities and assembly venues such as stadiums, sports halls, shops, theatres. Ventilation is the process of "changing" or replacing air in any space to provide high indoor air quality (to replenish oxygen, or remove moisture, odors, smoke, heat, dust, airborne bacteria, and carbon dioxide). This air exchange removes unpleasant smells and excessive moisture, introduces outside air, and prevents stagnation of the interior air. For this purpose, natural or mechanical methods can be used. Natural ventilation may be achieved with openable windows or trickle vents when the spaces to ventilate are small and there will be architectural conditions conducive to air circulation. Mechanical ventilation is achieved with use of air-conditioning systems. The ventilation rate, for buildings, is normally expressed by the volumetric flowrate of outside air being introduced to the building. For residential buildings, which mostly rely on infiltration for meeting their ventilation needs, the common ventilation rate measure is the number of times the whole interior volume of air is replaced per hour, and is called air changes per hour $(\mathrm{ACH})$. During the winter, ACH may range from 0.50 to 0.41 in a tightly insulated house to 1.11 to 1.47 in a loosely insulated house [14].

Such information is now particularly relevant to general hygiene and compliance with ventilation standards will be assessed more and more strictly.

There is also the concept of respiratory hygiene which means wearing masks, coughing in the elbow or covering mouth and nose with a tissue when coughing and sneezing. The tissue should be thrown to the bin once used. As well as discussed above, hand hygiene is part of the protection against spread of airborne diseases.

However, protection alone is not enough in the event of a possible exposure to infection or asymptomatic infection, it requires one more action to limit the spread of airborne diseases. Sometimes it is necessary to isolate infected people or those who are suspected of having a highly contagious infection.

Quarantine is a state, period, or place of isolation in which people or animals that have arrived from elsewhere or been exposed to infectious or contagious disease are placed. The word "quarantine" originates from quarantena, the Venetian language form, meaning "forty days". This is due to the 40-day isolation of ships and people practiced as a measure of disease prevention related to the plague. Between 1348 and 1359, the plague killed an estimated 30\% of Europe's population. So the ships could not enter the harbor and spent forty days at bay. In common language we still 
use the phrase "to keep someone at bay" which means to prevent someone from moving closer [15]. In the $19^{\text {th }}$ century yellow fever was imported by sailors to Spain from subtropical zone, and cholera in 1831 from Asia. In 1918-1919 the Spanish influenza pandemic killed an estimated 100 million people.

In the era of SARS-CoV-2 pandemic we look for the most effective ways to prevent the spread of infection (with hygiene and isolation of sick and suspected subjects). There is a done a great effort in order to identify the most effective methods of treatment and immunization.

\section{Conclusions}

The term "hygiene" returns to general public awareness again.

Hand hygiene became mandatory procedure for all healthcare workers and members of the public worldwide. The lack of strict adherence to hygienic procedures, overpopulation, intensive people migration and lowered herd immunity increase the risk of epidemic of infectious diseases like SARS-Cov-2. What is worth to be emphasized, the hygiene is a sign of professionalism and respect for the patients, ourselves and our co-workers.

The author declares no conflict of interest.

\section{References}

1. Semmelweis IP. Die Aetiologie, der Begriff und die Prophylaxe des Kindbettfiebers. Pest, Wien und Leipzig: C. A. Hartleben's Verlags-Expedition 1861

2. World Health Organization. WHO Guidelines on Hand Hygiene in Health Care. World Health Organization 2009.

3. Wojtyniak B, Goryński P. Sytuacja zdrowotna ludności Polski i jej uwarunkowania. Instytut Zdrowia Publicznego - Państwowy Zakład Higieny, Warszawa 2018.

4. Ezzati M, Riboli E. Behavioral and dietary risk factors for noncommunicable diseases. N Engl J Med 2013; 369: 954-964, DOI: 10.1056/NEJMra1203528.

5. Vincent JL. Nosocomial infections in adult intensive care units. Lancet 2003; 361: 2068-2077, DOI: 10.1016/S01406736(03)13644-6.
6. Klevens R, Edwards JR, Richards CL, et al. Estimating health care-associated infections and deaths in U.S. hospitals, 2002. Public Health Rep 2007; 122: 160-166, DOI: 10.1177/003335 490712200205.

7. Larson EL, Eke PI, Wilder MP, Laughon BE. Quantity of soap as a variable in handwashing. Infect Control 1987; 8: 371-375, DOI: 10.1017/s0195941700067436.

8. Ahmed J, Malik F, Memon ZA, et al. Compliance and Knowledge of Healthcare Workers Regarding Hand Hygiene and Use of Disinfectants: A Study Based in Karachi. Cureus 2020; 12: e7036, DOI: 10.7759/cureus.7036.

9. Sax H, Uçkay I, Richet H, Allegranzi B. Determinants of good adherence to hand hygiene among healthcare workers who have extensive exposure to hand hygiene campaigns. Infect Control Hosp Epidemiol 2007; 28: 1267-1274, DOI: $10.1086 / 521663$.

10. Kac G, Podglajen I, Gueneret M, et al. Microbiological evaluation of two hand hygiene procedures achieved by healthcare workers during routine patient care: a randomized study. J Hosp Infect 2005; 60: 32-39, DOI: 10.1016/j.jhin.2004.10.014.

11. Gorou E, Loyeau S, Legrand P, et al. Efficacy of handrubbing with alcohol based solution versus standard handwashing with antiseptic soap: randomised clinical trial. BMJ 2002; 325: 362, DOI: $10.1136 / \mathrm{bmj} .325 .7360 .362$.

12. Chow A, Arah OA, Chan SP, et al. Alcohol handrubbing and chlorhexidine handwashing protocols for routine hospital practice: a randomized clinical trial of protocol efficacy and time effectiveness. Am J Infect Control 2012; 40: 800-805, DOI: 10.1016/j.ajic.2011.10.005.

13. Pires D, Soule H, Bellissimo-Rodrigues F, et al. Antibacterial efficacy of handrubbing for 15 versus 30 seconds: EN 1500-based randomized experimental study with different loads of Staphylococcus aureus and Escherichia coli. Clin Microbiol Infect 2019; 25: 851-856, DOI: 10.1016/j.cmi.2018.10.012.

14. ANSI/ASHRAE Standard 62.2-2013: Ventilation and Acceptable Indoor Air Quality in Low-Rise Residential Buildings. Atlanta, GA: American Society of Heating, Refrigerating and Air-Conditioning Engineers, 2013.

15. Cambridge Dictionary, https://dictionary.cambridge.org/pl/ dictionary/english/ (access: 25.03.2020). 